\title{
TOWARDS PRACTICAL IMPLEMENTATION OF TIME OPTIMAL TRAJECTORIES FOR UNDERWATER VEHICLES
}

\author{
M. Chyba; T. Haberkorn; R.N. Smith; S. Zhao and S.K. Choi ${ }^{\ddagger}$ \\ *Department of Mathematics \\ 'Department of Ocean \& Resources Engineering \\ Autonomous Systems Laboratory \\ University of Hawaii \\ Honolulu, Hawaii 96822 \\ Email: mchyba@math.hawaii.edu, haberkor@math.hawaii.edu, \\ ryan@ore.hawaii.edu, zhao@wiliki.eng.hawaii.edu, schoi@maseinc.com
}

\begin{abstract}
In this paper, we are concerned with the practical implementation of time optimal numerical techniques on underwater vehicles. We briefly introduce the model of underwater vehicle we consider and present the parameters for the test bed ODIN (Omni-Directional Intelligent Navigator). Then we explain the numerical method used to obtain time optimal trajectories with a structure suitable for the implementation. We follow this with a discussion on the modifications to be made considering the characteristics of ODIN. Finally, we illustrate our computations with some experimental results.
\end{abstract}

\section{INTRODUCTION}

The use of underwater vehicles is expanding in every area of ocean science. Such vehicles are used by oceanographers, archaeologists, geologists, ocean engineers, and many others. These vehicles are designed to be agile versatile, and robust. The autonomy of such a vehicle only adds to the versatility and implementability of the vehicle. Much research has gone into making autonomous vehicles that travel underwater and perform a multitude of tasks far surpassing the abilities of human divers and small human-driven submarines. Tasks range from gathering ocean samples to 3-D mapping of underwater caverns and never before seen areas. Also, these vehicles can dive deeper and remain at depth longer without the risk of human life.
Although these autonomous robots are quite sophisticated, they are still confronted with the task of path planning for each of their missions. This task is a crucial one for the vehicle, and has yet to be fully investigated. Currently, most hydrodynamic models are estimated or use values from the literature. Also, vehicle controllers are generally model-free due to the poorly known nonlinear hydrodynamic model. Moreover, the motion planning of a non-linear system is a very difficult problem. However, we can expect that modeling of underwater systems will advance due to the significance and importance of AUVs in ocean science.

Anticipating this advance, our controllers will require more efficient algorithms to accurately tune the vehicles performance. With this in mind, we work to create algorithms to assist the controller in trajectory planning. However, our intent is not only to plan any path, but to plan a path which reduces some criterion, such as time or energy. Since, given multiple ways to do the same thing, we always seek the way to do it as efficiently as possible with respect to some metric. AUV users are always looking for a vehicle that can stay down longer, or finish the transects faster, or stabilize at a deeper depth, etc. With this motivation, we use this paper to examine the implementation of optimal control strategies, for the time domain, on an existing AUV testbed.

First we discuss the model we are using for our controlled mechanical system as well as the AUV we are considering and some of its main characteristics. The second part is dedicated presenting a method to compute implementable strategies derived from 
time optimal trajectories. These strategies are easier to implement than their time optimal counterparts, but are not far from being time optimal themselves. The third section deals with an adaptation of the computed strategies that account for the characteristics of ODIN. Finally, we illustrate our method with a field experiment on ODIN.

\section{THE MODEL}

We consider the general equations of motion for marine vehicles in 6 degrees of freedom and express them as a controlled mechanical system. We denote by $\eta=(x, y, z, \phi, \theta, \psi)^{t}$ the position and orientation of the vehicle in the earth-fixed reference frame, where $(\phi, \theta, \psi)$ are the classical Euler angles. The velocities $v=(u, v, w, p, q, r)^{t}$ are taken with respect to the body-fixed frame (see Figure 1).



With these notations, the equations of motion can be written as, see [1]:

$$
\begin{aligned}
& \dot{\eta}=J(\eta) v \\
& \dot{v}=M^{-1}(\tau-[C(v)+D(v)] v-g(\eta))
\end{aligned}
$$

where $J(\eta)$ is the transformation matrix from the body-fixed to the earth-fixed coordinate systems. $M$ is the inertia matrix for the vehicle. The matrix $C$ accounts for the Coriolis and centrifugal forces on the vehicle, and it is assumed to be skew-symmetric. The matrix $D$ represents the damping forces. The column vector $g$ represents the restoring forces and moments due to gravity and buoyancy. Lastly, $\tau$ is the control.

We assume that the thrusters have limited power, and incorporate this by defining $\tau$ as follows:

$$
\tau \in \mathcal{U}_{b}=\left\{\tau \in \mathbb{R}^{6} \mid \alpha_{i} \leq \tau_{i} \leq \beta_{i}, i=1, \cdots, 6\right\}
$$

where $\alpha_{i}<0<\beta_{i}$.

We use this control domain, $\mathcal{U}_{b}$ when applying optimal control theory in order to find time optimal thrust strategies. We find that such strategies are bang-singular, see [2-5]. This means that the thrust consists of a concatenation of maximum, or minimum, constant thrust arcs and continuous thrust arcs. This control domain consists of a thrust vector over $\mathbb{R}^{6}$ respective to the movement of the vehicle in $6 \mathrm{DOF}$.

The testbed vehicle, ODIN, used for the experiments has a spherical hull that is $65 \mathrm{~cm}$ in diameter. (see Figure 3 )

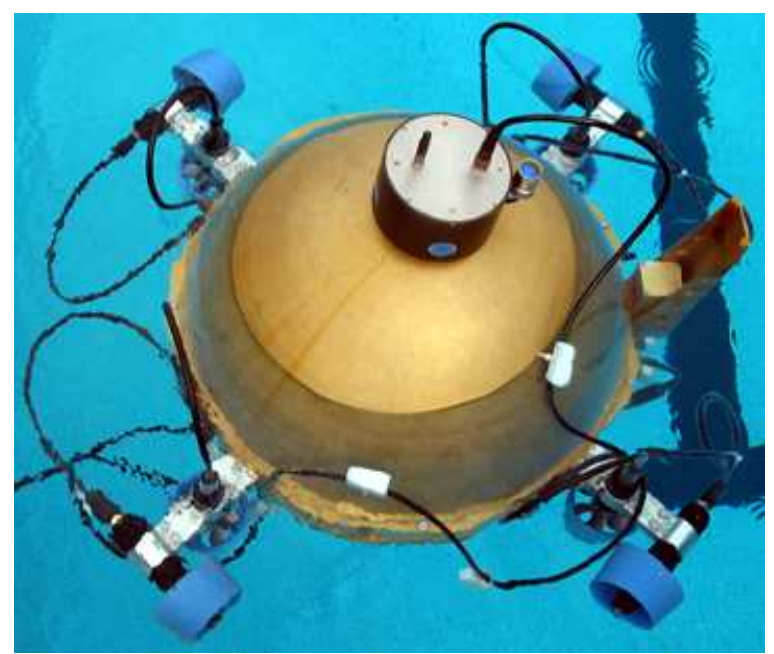

Figure 2. ODIN in the pool as seen from above.

This sphere is constructed from an aluminum alloy to prevent corrosion. Eight thrusters are attached to the sphere via four fabricated mounts, each holding two thrusters. These thrusters are evenly distributed around the sphere with four vertical and four horizontal, see Figure 2. A fixed "arm" is attached to ODIN to denote the forward direction as well as allow for the attachment of devices such as cameras, hooks, or sampling devices. Fully assembled, ODIN weighs $120 \mathrm{~kg}$ and is positively buoyant by $4 \mathrm{~kg}$. ODIN is capable of moving in 6 DOF from either a tethered or autonomous mode. For our experiments, ODIN is tethered, but only to send commands to be run in an autonomous mode. This setup allows for multiple tests to be conducted without removing ODIN from the water to upload mission sorties. ODIN's internal CPU is based on a Motorola 68030. Also contained within ODIN is an IMU, leakage sensor, and heat sensors. ODIN is equipped with eight sonar sensors for positioning; there are four front, one left, one right, and one rear facing sonars. There is a pressure sensor to determine depth of the vehicle. The IGW (Integrated Graphic Workstation) is a 3-dimensional, real-time graphic platform to monitor and dry-test AUVs. The IGW is composed of a Silicon Graphics Onyx RE2, a Tektronix 29Pex terminal, and Wavefront's Advanced Visualizer and Data Visualizer softwares. The communication from ODIN to IGW is through a RS232c serial line via laptop. This setup brings mobility to ODIN while 


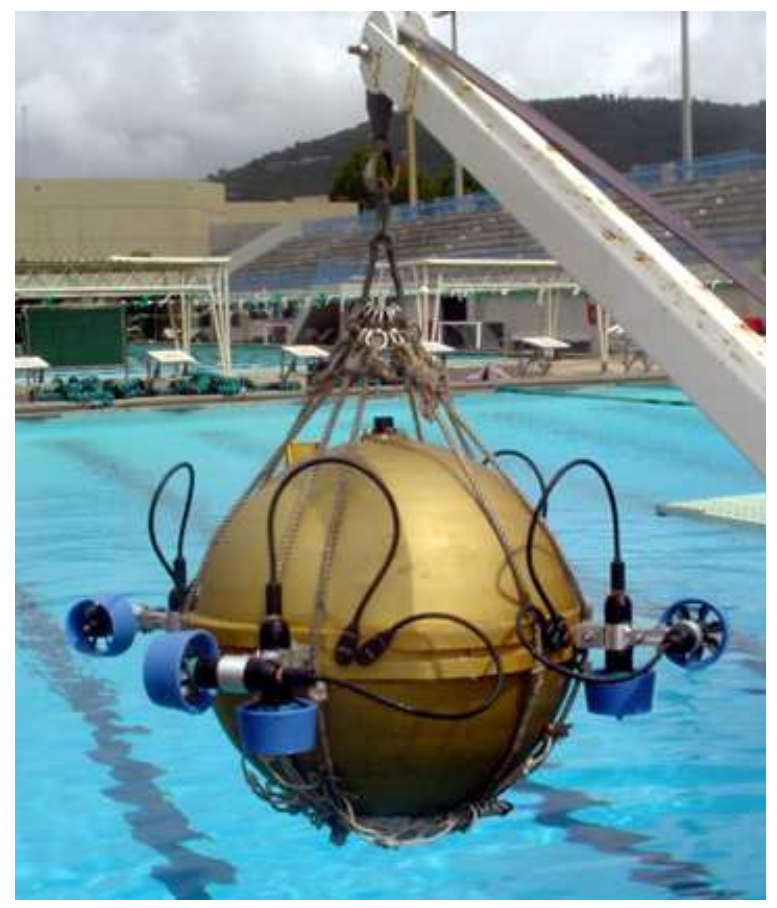

Figure 3. ODIN being lowered into the pool for testing.

the IGW remains stationary in the laboratory. The entire system is compact and allows for easy transport to and from the testing site along with the ability to alter mission profiles on the fly during testing. Unique to ODIN's construction is the control from an eight dimensional thrust to move in 6 DOF. This construction puts redundancy into the system in case of thruster failure. ODIN is able to operate in an "underactuated" condition if necessary. To compute the 6 dimensional thrust $\tau$ resulting from the 8 dimensional thrust $\gamma$ (from the thrusters), we need to apply a linear transformation to $\gamma$. Since the 4 vertical thrusters only contribute to the Heave, Pitch and Roll motion (and the 4 horizontal thrusters to the 3 other directions), we can decouple the transformation into two linear transformations from $\mathbb{R}^{4}$ to $\mathbb{R}^{3}$. The matrix representation of those two linear transformations are called $T C M_{\text {ver }}$ and $T C M_{\text {hor }}$, for the vertical and horizontal Thruster Control Matrix respectively. For ODIN, these are as follows:

$$
\begin{gathered}
T C M_{\mathrm{ver}}=\left[\begin{array}{cccc}
-1 & -1 & -1 & -1 \\
-0.2676 & -0.2676 & 0.2676 & 0.2676 \\
-0.2676 & 0.2676 & 0.2676 & -0.2676
\end{array}\right] \\
T C M_{\mathrm{hor}}=\left[\begin{array}{cccc}
-0.7071 & 0.7071 & 0.7071 & -0.7071 \\
0.7071 & 0.7071 & -0.7071 & -0.7071 \\
0.4801 & -0.4801 & 0.4801 & -0.4801
\end{array}\right]
\end{gathered}
$$

Since each thruster is independently powered, it is clear that the control domain on $\mathbb{R}^{8}$ is a box $\mathcal{G}$. So the real control domain of $\tau$ is the image of $\mathcal{G}$ through the linear transformation, we will call this image $\mathcal{U}_{r}$. Trying to fit $\mathcal{U}_{b}$ into $\mathcal{U}_{r}$ will prevent us from using a significant part of the real control domain, since $\mathcal{U}_{r}$ consists of two flat ellipsoids in $\mathbb{R}^{3}$. Hence, we also consider a model that uses 8 dimensional thrust instead of the resulting 6 dimensional thrust.

\section{Physical values}

For the model we use in the sequel, we assume the following shape of the inertia matrix, $M$ :

$$
M=\left[\begin{array}{cccccc}
3 / 2 m & 0 & 0 & 0 & m z_{G} & -m y_{G} \\
0 & 3 / 2 m & 0 & -m z_{G} & 0 & m x_{G} \\
0 & 0 & 3 / 2 m & m y_{G} & -m x_{G} & 0 \\
0 & -m z_{G} & m y_{G} & I_{x} & -I_{x y} & -I_{x z} \\
m z_{G} & 0 & -m x_{G} & -I_{x y} & I_{y} & -I_{y z} \\
-m y_{G} & m x_{G} & 0 & -I_{x z} & -I_{y z} & I_{z}
\end{array}\right]
$$

Where $m=120.9091 \mathrm{~kg}$ is the mass of ODIN, $\left(x_{G}, y_{G}, z_{G}\right)=$ $(0,0,5.64 e-4)$ (meters) is the center of gravity (in the bodyfixed frame), and $I$. and $I_{\text {.. }}$ are inertia factors $\left(I_{x}=6.8 \mathrm{~kg} . \mathrm{m}, I_{y}=\right.$ $\left.7.2 \mathrm{~kg} . \mathrm{m}, I_{z}=9.1 \mathrm{~kg} . \mathrm{m}, I_{y z}=I_{x z}=I_{y z}=0\right)$. The restoring forces and moment vector $g(\eta)$ is taken to be:

$g(\eta)=\left[\begin{array}{c}\left(W_{g}-B\right) \sin \theta \\ \left(-W_{g}+B\right) \cos \theta \sin \phi \\ \left(-W_{g}+B\right) \cos \theta \cos \phi \\ \left(-y_{G} W_{g}+y_{B} B\right) \cos \theta \cos \phi+\left(z_{G} W_{g}-z_{B} B\right) \cos \theta \sin \phi \\ \left(z_{G} W_{g}-z_{B} B\right) \sin \theta+\left(x_{G} W_{g}-x_{B} B\right) \cos \theta \cos \phi \\ \left(-x_{G} W_{g}+x_{B} B\right) \cos \theta \sin \phi-\left(y_{G} W_{g}-y_{B} B\right) \sin \theta\end{array}\right]$

Where $\left(x_{B}, y_{B}, z_{B}\right)=(0,0,8.1 e-4)$ (meters) stands for the center of buoyancy, $W_{g}$ is the weight of the vehicle, and $B=$ $W_{g}+38$ (Newton) is the buoyancy force on the vehicle. The damping forces $D(v)$ are of the form $-\operatorname{diag}\left(X_{u}+X_{u u}|u|, Y_{v}+\right.$ $\left.Y_{v v}|v|, Z_{w}+Z_{w w}|w|, K_{p}+K_{p p}|p|, M_{q}+M_{q q}|q|, N_{r}+N_{r r}|r|\right)$ where $\left(X_{u}, X_{u u}\right)(=(0,-150)),\left(Y_{v}, Y_{v v}\right)(=(0,-150))$, and $\left(Z_{w}, Z_{w w}\right)$ $(=(0,-150))$ are the drag coefficients for pure surge, sway, and heave, respectively. And, $\left(K_{p}, K_{p p}\right)(=(-140,-180))$, $\left(M_{q}, M_{q q}\right)(=(-140,-180)),\left(N_{r}, N_{r r}\right)(=(-140,-180))$ are the drag coefficients for pure roll, pitch, and yaw, respectively.

The coriolis matrix $C$ is of the form: 


$$
\left[\begin{array}{ccc}
0 & 0 & 0 \\
0 & 0 & 0 \\
0 & 0 & 0 \\
-m\left(x_{G} q+z_{G} r\right) & m\left(y_{G} p+w\right)-Z_{w d} w & m\left(z_{G} p-v\right)+Y_{v d} v \\
m\left(x_{G} q-w\right)+Z_{w d} w & -m\left(z_{G} r+x_{G} p\right) & m\left(u+z_{G} q\right)-X_{u d} u \\
m\left(x_{G} r+v\right)-Y_{v d} v & m\left(y_{G} r-u\right)+X_{u d} u & -m\left(x_{G} p+y_{G} q\right) \\
-m\left(y_{G} q+z_{G} r\right) & m\left(w-x_{G} q\right)-Z_{w d} w & m\left(z_{G} p-v\right)+Y_{v d} v \\
Z_{w d} w-m\left(y_{G} p+w\right) & -m\left(z_{G} r+x_{G} p\right) & m\left(u-y_{G} r\right)-X_{u d} u \\
m\left(v-z_{G} p\right)-Y_{v d} v & X_{u d} u-m\left(u+z_{G} q\right) & m\left(x_{G} p+y_{G} q\right) \\
0 & C_{4,5} & C_{4,6} \\
C_{5,4} & 0 & C_{5,6} \\
C_{6,4} & C_{6,5} & 0
\end{array}\right]
$$

Where $X_{u d}=Y_{v d}=Z_{w d}=-m / 2$ and $K_{p d}=M_{q d}=N_{r d}=0$ and the coefficients $C_{\ldots}$ are:

$$
\begin{aligned}
& C_{4,5}=I_{z} r-I_{y z} q-I_{x z} p-N_{r d} r, \quad C_{4,6}=I_{y z} r+I_{x y} p-I_{y} q+M_{q d} q \\
& C_{5,4}=I_{y z} q+I_{x z} p-I_{z} r+N_{r d} r, \quad C_{5,6}=I_{x z} r-I_{x y} q+I_{x} p-K_{p d} p \\
& C_{6,4}=I_{y} q-I_{y z} r-I_{x y} p-M_{q d} q, C_{6,5}=I_{x y} q-I_{x} p-I_{x z} r+K_{p d} p
\end{aligned}
$$

The two control domains are taken as follows (Newton):

$$
\begin{array}{r}
\mathcal{G}=[-14.2791,6.3925]^{8} \\
\mathcal{U}_{b}=[-8.3,8.3]^{2} \times[-10,44] \times[-2.25,2.25]^{2} \times[-1,1]
\end{array}
$$

where the main restriction used to choose $\mathcal{U}_{b}$ is that $\tau_{3}$ is able to counter balance the buoyancy of the vehicle.

\section{DESIGNING TIME OPTIMAL TRAJECTORIES}

We are interested in finding time optimal trajectories which steer our AUV from an initial state $\chi_{0}=\left(\eta_{0}, v_{0}\right)$, to a final state $\chi_{f}=\left(\eta_{f}, v_{f}\right)$, usually both at rest $\left(v_{0}=v_{f}=0\right)$. These optimal trajectories are computed with respect to our dynamic (1). The following is the optimal control problem that we set out to solve:

$$
(O C P) u_{b}\left\{\begin{array}{l}
\min t^{f} \\
\dot{\eta}=J(\eta) v \\
\dot{v}=M^{-1}(\tau-[C(v)+D(v)] v-g(\eta)) \\
\tau \in \mathcal{U}_{b} \\
(\eta(0), v(0))=\chi_{0} \\
\left(\eta\left(t^{f}\right), v\left(t^{f}\right)\right)=\chi_{f}
\end{array}\right.
$$

We will only consider solving $(O C P)_{\mathfrak{U}_{b}}$ since the extension to $(O C P)_{\mathcal{G}}$ is done similarly.
As explained in [2-5], and according to the Maximum Principle [6], a thrust strategy solution of $(O C P) u_{b}$ is a concatenation of constant thrust arcs with maximum magnitude (called bang arcs) along with continuous thrust arcs (called singular arcs). A time when bang arcs change value or change to singular arcs is referred to as a switching time. Another possibility is that the optimal thrust strategy includes some chattering at a junction between singular and bang arcs, see [7]. Chattering occurs when there are infinite switchings from one bang arc value to the other. In this paper, we only work with strategies that have no chattering.

One way to solve $(O C P) u_{b}$ is to discretize it along a time grid, then rewrite it as a nonlinear optimization problem whose unknowns are the discretized states and controls at each time step. After this, one can use a nonlinear large-scale optimization solver to find a solution of the discretized problem. Using the modeling language $A M P L$, [8], along with the solver IpOpt, [9], we get the kind of thrust strategy shown in Figure 4 (here for $\chi_{0}=0$, $\eta_{f}=(3,3,1, \cdots, 0)$ and $\left.v_{f}=0\right)$. In this figure, we see that

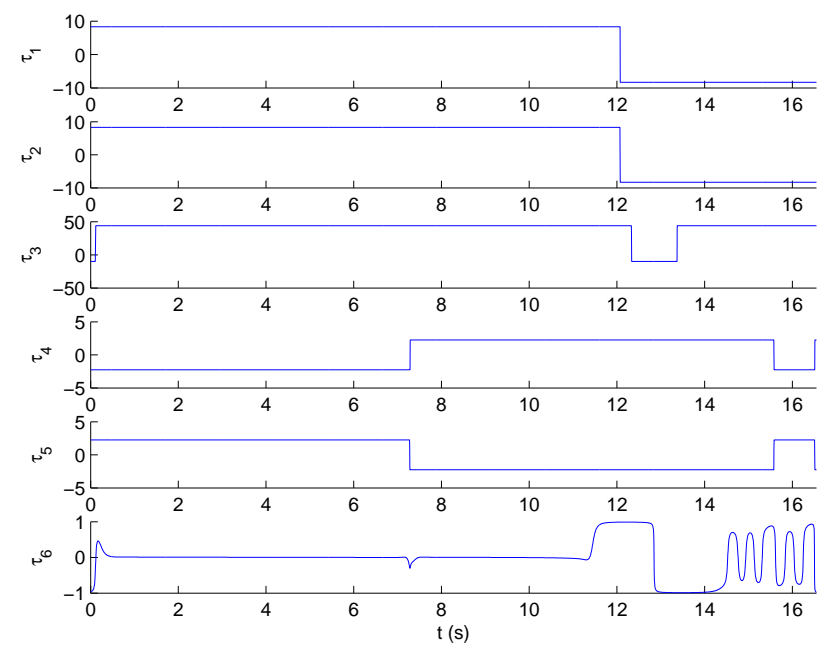

Figure 4. Time optimal thrust strategies obtained by discretization of $(O C P)_{u_{b}}$

we have only bang controls with an exception in the $\tau_{6}$ control. This control is almost singular. The final time of this solution is $t_{\text {min }}^{f} \approx 16.537 \mathrm{~s}$. This thrust strategy raises two problems concerning practical implementation onto a testbed AUV. The first problem is the discretization of $(O C P) u_{b}$ introduces inaccuracy. This comes from using a fixed and low order integration scheme to integrate (1). A second issue is partial singularity in the $\tau_{6}$ control. The singularity is difficult to implement since an evolving thrust arc must be applied in $\tau_{6}$, as opposed to a simple constant thrust arc. Furthermore, the optimization problem has a large 
number of unknowns, and can be very time consuming to solve. To overcome the above problems, we propose a method inspired from $[10,11]$. This method looks for a solution that is optimal with respect to a given number of switching times. So, we fix the number of switching times and find an optimal trajectory with that many switchings. This allows us to rewrite $(O C P) u_{b}$ as an optimization problem, called $(N L P)_{u_{b}}$, whose unknowns are the values of the switching times as well as the values of the constant thrust arcs. The only nonlinear constraints of $(N L P) \mathfrak{u}_{b}$ are the conditions on the final configurations. This $(N L P) \mathcal{U}_{b}$ has some serious advantages over the $(O C P) u_{b}$. Indeed, $(N L P) u_{b}$ has a small number of parameters, usually not more than a hundred, while $(O C P)_{\mathfrak{u}_{b}}$ easily surpasses tens of thousands. This reduction, on the number of parameters, directly implies an increase in solving speed. Solving the first nonlinear optimization problem takes approximatly $30 \mathrm{~min}$ (all refinements included), while solving $(N L P)_{\mathcal{U}_{b}}$ takes around $10 \mathrm{secs}$. Moreover, we can use any integration scheme to integrate (1). Thus, the accuracy of the solution will be a better match to our model. Of course, $(O C P) u_{b}$ has an advantage over $(N L P) u_{b}$ since the later looks for a solution over a narrower range of possible thrust strategies. So, $(O C P) u_{b}$ will always be expected to have a smaller final time. However, if we compare the final times from the two different approaches, we see that they are not that different. Also, as seen in Table 1, as we use more switching times, the final time approaches the minimum time for the problem.

\begin{tabular}{c|c} 
\# of Switching Times & $t^{f}(\mathrm{~s})$ \\
\hline optimal & 16.537 \\
1 & 18.555 \\
2 & 17.784 \\
3 & 16.887 \\
4 & 16.618 \\
5 & 16.589
\end{tabular}

Table 1. Final time with respect to number of Switching Times.

When using only 2 switching times, problem $(N L P) u_{b}$ yields a final time $t^{f} \approx 17.784 \mathrm{~s}$ versus $16.537 \mathrm{~s}$ for $(O C P) u_{b}$. This is only a $7.5 \%$ difference, and the corresponding thrust strategy is easier to implement, as shown on Figure 5. Thus we get an accurate integration of the model, and an easier way to compute the thrust arcs. As it is expected, this thrust strategy is bang in $\tau_{1}$ and $\tau_{2}$ since it uses the full power of these controls. The other values of the constant thrust arcs are not of maximum magnitude. These are adjusted in such a way that the most

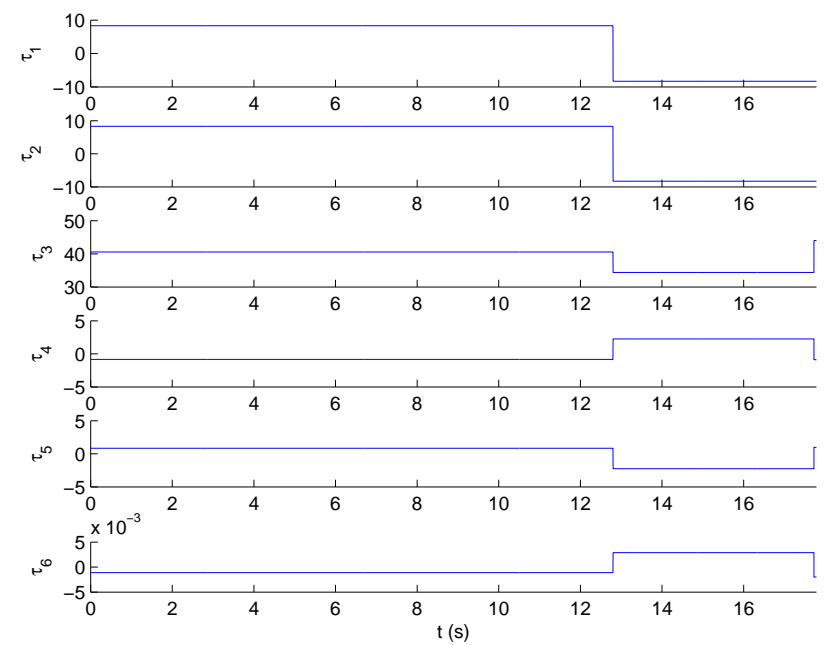

Figure 5. Thrust strategy for 2 switching times, $\chi_{0}=0, \eta_{f}=$ $(3,3,1,0,0,0)$ and $v_{0}=0$.

difficult parts of the transfer (the Surge and Sway motion) are done as fast as possible. Note that the $\tau_{6}$ control is almost zero along the trajectory which implies that the Yaw orientation will stay very close to zero.

Let us now move to the next step which is a practical implementation of this thrust strategy or, to be more precise, an adaptation of it.

\section{ADAPTATION OF TRAJECTORIES}

Even if the previous thrust strategy is fairly simple, it is illusionary to believe that a straightforward open loop implementation on a testbed AUV, will yield good results. Indeed, for such an implementation to be successful we need our model to be very accurate; we know this is not the case, since we still have to develop an acceptable parameter estimation method. Moreover, even if the number of switchings is small, the discontinuities implied by them will give rise to inaccuracies in our thruster power. It is probable that we will also need to improve our thruster modeling. For the moment we will treat these two issues indirectly by considering the following.

Since our models (dynamic and thruster) are probably too inaccurate, we will only implement the open loop thrust strategy in the components that seem less sensitive to the overall behavior of the vehicle. Between translational and orientation directions, the translational are less sensitive. This is particularly verified in the Yaw because of the spherical shape of the vehicle, and since the center of gravity and buoyancy lie on the same vertical line. We choose to apply an open thrust strategy in $\tau_{1}, \tau_{2}$ and $\tau_{3}$ and to compute closed loop controls $\tau_{4}, \tau_{5}$ and $\tau_{6}$. Since, a misapplied force in $\tau_{1}, \tau_{2}$ or $\tau_{3}$ has a resistance of buoyancy force or 
drag, which seems to have more effect than on those in $\tau_{4}, \tau_{5}$ and $\tau_{6}$. The closed loop controls are computed so that the orientation in Pitch, Roll and Yaw tracks the open loop trajectory. The computation is performed using a PID controller.

Remark 1. Computing $\tau_{4}$ to correct the Pitch, $\tau_{5}$ to correct the Roll and $\tau_{6}$ to correct the Yaw implies that we assume the system to be decoupled, i.e. the inertia matrix to be diagonal. This is an approximation that can be considered valid since the center of gravity of our vehicle is nearly coincident with the origin of our body-fixed frame.

The theoretical trajectories corresponding to the thrust strategies shown on Figures 5 and 4, are shown on Figure 6, but only for $\eta$ (position/orientation) and not for $v$ (velocity).



Figure 6. Time optimal trajectory (continuous line) and trajectory for 2 switching times (dashed line), $\chi_{0}=0, \eta_{f}=$ $(3,3,1,0,0,0)$.

One can see that the two trajectories don't differ much, except maybe in the evolution of $\psi$ which is nearly zero.

The Pitch and Roll do not vary much. Their magnitude is contained in a 0.1 radian (less than $6^{\circ}$ ) neighborhood of zero. As a consequence, we see that the Yaw is almost zero along the trajectory.

Remark 2. This nearly zero Yaw might still be hard to track and/or stabilize due to the non-symmetry of the 4 horizontal thrusters. An application of thrust in $\tau_{1,2}$ implies a thrust in $\tau_{1,2,6}$ from the un-symmetric thrusters. Hence, the tracking is necessary.

Remark 3. Using a thrust strategy and a trajectory obtained by the computation with $\mathcal{U}_{b}$ instead of $\mathcal{G}$ unavoidably yields a greater final time. But, the advantage with the $\mathcal{U}_{b}$ control domain is significantly reducing the risk of exceeding the thrusters limitations. Indeed, the closed loop computation of $\tau_{4,5,6}$ may yield over-excessive thrust.

The next section presents one of our field experiments. These experiments were conducted in the diving well of the Duke Kahanamoku Swimming Complex at the University of Hawaii at Manoa.

\section{FIELD EXPERIMENTS}

The thrust strategy applied results in a final configuration at rest $\eta_{f}=(3,3,0,0.1,0.1,0.25)$. We begin by considering a thrust strategy with only one switching time, as a beginning point. This final configuration is chosen so that the AUV evolves in every Euler angle. We choose this strategy to test the efficiency of the tracking. Orientation is updated every $990 \mathrm{~ms}$, and we update the open loop controls $\tau_{1,2,3}$ every $30 \mathrm{~ms}$.

ODIN's sonar sensors were inoperable at the time of the tests, which limited our knowledge of his $\mathrm{x}, \mathrm{y}$-position. This was solved by video taping the experiments from the $10 \mathrm{~m}$ diving platform. The diving platform offered a position to view ODIN from directly above. Using a video tracking technique, we are able to track ODIN in the $x-y$ plane with accuracy about $5 \mathrm{~cm}$. This is not a real time utility, but the nature of the experiment does not require real time feedback. Also, since a proper $x-y$ trajectory with significant orientation evolution is un-realistic, we show the evolution of ODIN in figure 7 in depth $z$, and in the orientations $\phi, \theta$ and $\psi$.

The experimental resultant evolution of ODIN is given by the continuous line. The dotted line represents the desired trajectory, where all controls are computed in an open loop method. The dashed line corresponds to the theoretical trajectory assuming a perfect hydrodynamic model and Roll, Pitch and Yaw computed in a closed loop method. In particular, $\tau_{1,2,3}$ are computed in open loop and $\tau_{4,5,6}$ in closed loop.

First, let us consider the orientation tracking. The theoretical and experimental trajectories have the same trends in both the $\phi$ and $\theta$ angles, but there is a slight shift. The greatest difference in $\theta$ is almost 0.1 radian $\left(\approx 6.3^{\circ}\right)$, which is the difference between the final and the initial prescribed value. Seeing the evolution of $\psi$, may lead one to believe that the experiment is bad. However, note that the $\tau_{1,2}$ controls share the same horizontal thrusters as $\tau_{6}$. Thus, any non symmetry in the behavior of those thrusters when computing a $\tau_{1,2}$ control will result in a parasite $\tau_{6}$ control. This parasite $\tau_{6}$ control may not be easily compensated for in the controller. This is due to the original design for the controller being for stability tasks rather than for tracking tasks. The depth evolution is promising, since we lost less than $30 \mathrm{~cm}$ of depth during this $30 \mathrm{~s}$ experiment. Of course, the good results in the depth is directly related to the effectiveness of the orientation 

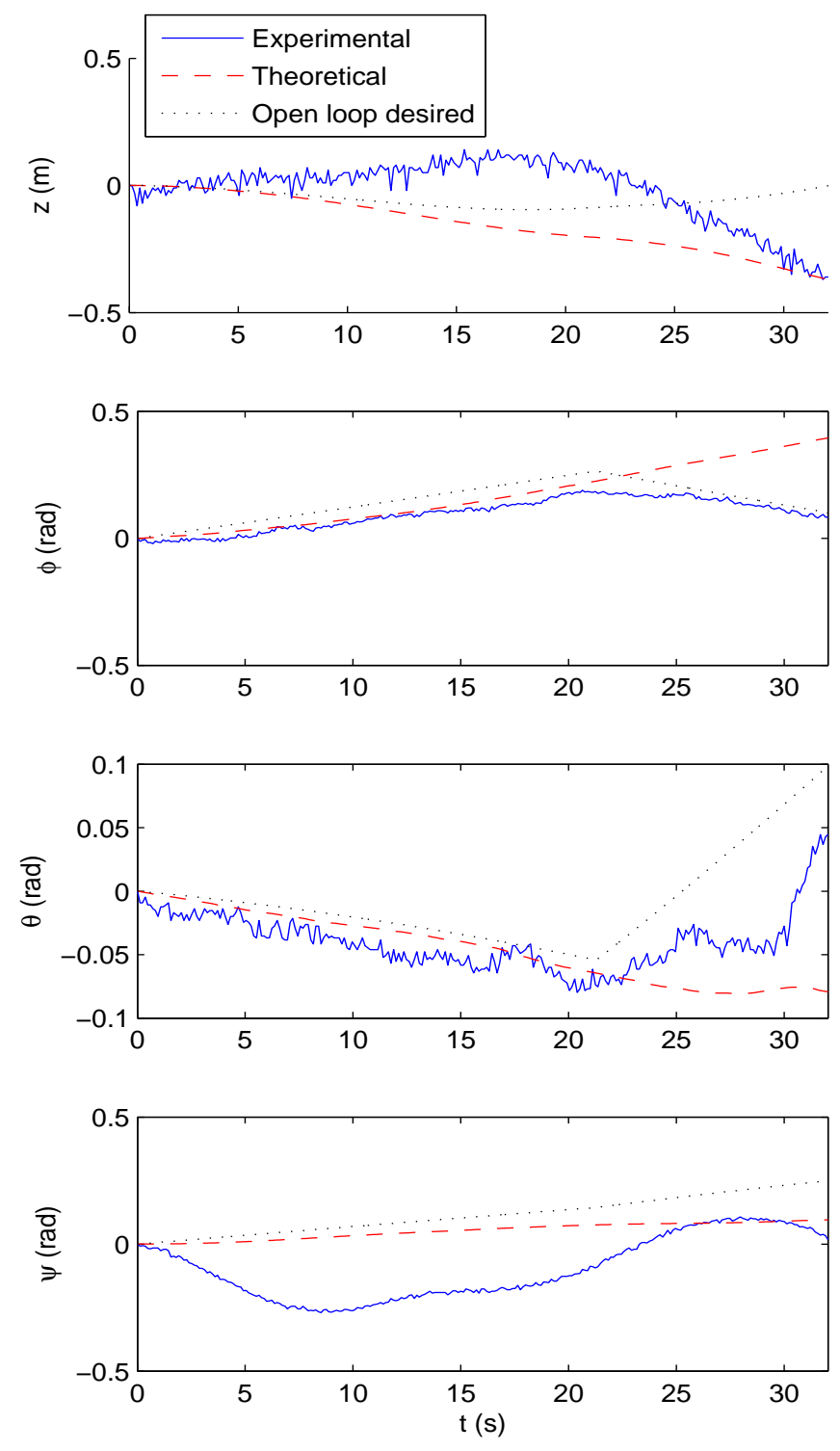

Figure 7. Comparison between the experimental behavior of ODIN and the theoretical ones.

tracking for this experiment. Note that the computed theoretical evolution which corresponds to the open loop thrust strategy $\left(\tau_{1,2,3}\right)$, and the thrust computed by ODIN's controllers $\left(\tau_{4,5,6}\right)$ do not match the experimental behavior of the vehicle. This indicates that our hydrodynamic model needs improvements. This only a confirms our expectations at the outset of this research. It is also necessary to improve our thruster model if we intend to pursue our mixed open/close loop experiments.

Our first experimental results give promise for the the theory behind the strategies computed. With improvements on our mod- els, and a closer study of the sensitivity of ODIN, with respect to hydrodynamic parameters; we expect to see increasingly better implementations of theoretical trajectories onto testbed AUV's.

\section{CONCLUSION}

In this paper, we present a numerical method to compute thrust strategies which are time optimal with respect to a fixed number of switching times. Such strategies are then modified to be more easily implemented on our test bed: ODIN. Lastly we present the results of our first experiments concerning such thrust strategies. These results seem promising, despite knowing their need of improvement. In order to perform those improvements, we will allocate some time to improve our thruster model as well as our dynamical model of ODIN. Such improvements need the application of accurate parameter estimation methods. Considering the sensitivity of the dynamical model to such parameters as the position of the center of gravity and of buoyancy, it is doubtful that such methods would be easily designed. In particular, assuming that the center of gravity and buoyancy of the vehicle are vertically aligned seems to be too restrictive and so the longitudinal position of those centers shouldn't be fixed during the parameter estimation. This implies that we cannot assume the system to be decoupled in our open loop theoretical model, contrary to assumptions made by most AUV on board controllers.

\section{REFERENCES}

[1] T.I.Fossen. "Guidance and control of ocean vehicles". Wiley, New York, 1994

[2] M. Chyba, N.E. Leonard and E.D. Sontag. "Singular trajectories in the multi-input time-optimal problem: Application to controlled mechanical systems". Journal on Dynamical and Control Systems 9(1):73-88, 2003.

[3] M. Chyba, N.E. Leonard and E.D. Sontag. "Optimality for underwater vehicles". In Proceedings of the 40th IEEE Conf. on Decision and Control, Orlando, 2001.

[4] M.Chyba, H. Maurer, H.J. Sussmann and G. Vossen. "Underwater Vehicles: The Minimum Time Problem”. In Proceedings of the 43th IEEE Conf. on Decision and Control, Bahamas, 2004.

[5] M. Chyba and T. Haberkorn. "Designing efficient trajectories for underwater vehicles using geometric control theory". Proceedings of the 24rd International Conference on Offshore Mechanics and Arctic Engineering, Greece, 12-17 June 2005.

[6] B. Bonnard and M. Chyba. "Singular Trajectories and their Role in Control Theory". Springer-Verlag, 2003.

[7] M. Chyba and T. Haberkorn. "Autonomous underwater vehicles: singular extremals and chattering". Proceedings of the 22nd IFIP conference on System modeling and Optimization, Turin, Italy, 18-22 July 2005. To appear. 
[8] R. Fourer, D.M. Gay and B.W. Kernighan. "AMPL: A Modeling Language for Mathematical Programming". Duxbury Press, Brooks-Cole Publishing Company, 1993.

[9] A. Waechter and L. T. Biegler. "On the Implementation of an Interior-Point Filter-Line Search Algorithm for Large-Scale Nonlinear Programming". Research Report RC 23149, IBM T.J. Watson Research Center, Yorktown, New-York.
[10] C.Y. Kaya and J.L. Noakes. "Computation method for timeoptimal switching control". Journal of Optimization Theory and Applications, 117(1):69-92, 2003.

[11] H. Maurer, C. Büskens, J-H.R. Kim and I.C. Kaya. "Optimization methods for numerical verification of second order sufficient conditions for bang-bang controls". Optimal Control Application and Methods, 26:129-156, 2005. 